\title{
Smart box-enabled product-service system for cloud logistics
}

Yingfeng Zhang, Sichao Liu, Yang Liu and Rui Li

The self-archived postprint version of this journal article is available at Linköping University Institutional Repository (DiVA):

http://urn.kb.se/resolve?urn=urn:nbn:se:liu:diva-133460

N.B.: When citing this work, cite the original publication.

This is an electronic version of an article published in:

Zhang, Y., Liu, S., Liu, Y., Li, R., (2016), Smart box-enabled product-service system for cloud logistics, International Journal of Production Research, 54(22), 6693-6706.

https://doi.org/10.1080/00207543.2015.1134840

Original publication available at:

https://doi.org/10.1080/00207543.2015.1134840

Copyright: Taylor and Francis

http://www.tandf.co.uk/journals/default.asp 


\title{
Smart box enabled product-service system for cloud logistics
}

\author{
Yingfeng Zhang ${ }^{1}$, Sichao Liu ${ }^{1}$, Yang $\mathrm{Liu}^{2}, \mathrm{Rui}^{1}{ }^{1}$ \\ ${ }^{1}$ Key Laboratory of Contemporary Design and Integrated Manufacturing Technology, \\ School of Mechanical Engineering, Northwestern Polytechnical University, Shaanxi, \\ China, 710072 \\ ${ }^{2}$ Department of Production, University of Vaasa, Vaasa, Finland
}

Corresponding Author: Yingfeng Zhang (zhangyf@nwpu.edu.cn)

Address: Room B509, Hang Kong Building, Department of Industrial Engineering, Northwestern Polytechnical University, Xi an, 710072, China 


\title{
Smart box enabled product-service system for cloud logistics
}

\begin{abstract}
Modern logistics takes significant progress and rapid developments with the prosperity of E-commerce, particularly in China. Typical challenges that logistics industry is facing now are composed by a lack of sharing, standard, cost-effective and environmental package, and efficient optimization method for logistics tasks distribution. As a result, it is difficult to implement green, sustainable logistics services. Three important technologies, Physical Internet (PI), Product Service System (PSS) and cloud computing (CC), are adopted and developed to address the above issues. PI is extended to design a world-standard green recyclable smart box that is used to encapsulate goods. Smart box enabled product service system is constructed to provide an innovative sustainable green logistics service, and high-quality packaging, as well as reduce logistics cost and environmental pollution. A real-time information driven logistics tasks optimization method is constructed by designing a cloud logistics platform based on cloud computing. On this platform, a hierarchical tree-structure network for customer orders is built up to achieve the order-box matching of function. Then, a distance clustering analysis algorithm is presented to group and form the optimal clustering results for all customer orders, and a real-time information driven optimization method for logistics orders is proposed to minimize the unused volume of containers. Finally, a case study is simulated to demonstrate the efficiency and feasibility of proposed cloud logistics optimization method.
\end{abstract}

Keywords: Physical Internet; Logistics; Smart Box; Product Service System; Cloud Computing

\section{Introduction}

With increasingly rapid development and implementation of the global E-commerce, the daily life and working manner of people has been significantly reformed, particularly online shopping, which results in extensive growth of modern logistics services. It is worth noted that Chinese E-commerce has taken the fast progress and made remarkable achievements in recent years. Emerging E-commerce suppliers change traditional manners of shopping thoroughly such as Alibaba 
(http://www.chinairn.com/news/20150130/15583829.shtml) and TAOBAO (http://www.ebrun.com/20150202/123162.shtml). It is widely adopted by the 80 's and 90 's youths for the convenient, flexible way of shopping. As a result, the relationship of E-commerce and modern logistics is more and more closely.

The statistics shows the number of express package delivery in China is about 14 billion in 2014 (http://data.stats.gov.cn/), and a large number of delivery requirements of logistics tasks accelerate small-sized and medium-sized logistics enterprises appearance. Here, logistics task indicates goods order that is required to be delivered to customers from suppliers according to the logistics information of tasks. These logistics enterprises usually have the following characteristics: small scale, separate operating, different operation pattern, and various delivery standard and process. Through deep investigations on current logistics enterprises and new requirements, the challenges that logistics industry is facing now are composed by strengthening information sharing and cooperation, reducing environmental pollution, and enhancing the loading rate while decreasing the logistics cost. Therefore, some research questions that logistics industry is facing are outlined as follows:

- How to design an overall architecture and solution to timely share the logistics information and dynamic cooperation among different logistics enterprises?

- How to design the new package way for encapsulating goods to deal with the disordered, ill-formed and huge environmental pollution problems?

- How to design a dynamic optimization method for logistics tasks distribution to improve the lower loading rate of vehicles and higher waste of fuels?

To address the above challenges and research questions, this research adopts three important technologies: Physical Internet, Product Service System, and Cloud 
Computing. PI has been conceptualized as an open global logistics system, leveraging interconnected supply networks through a standard set of collaborative protocols, modular containers, and smart interfaces for increased efficiency and sustainability (Montreuil, 2011). PI provides a technology support and infrastructure for constituting recyclable, world-standard smart green box and implementing smart box enabled cloud logistics services. PSS is a service-oriented sustainable product-service system, which integrates the products and services associated with their products (Williams, 2007). The concept of PSS is extended to logistics filed to establish a smart box enabled product-service system. This innovative business model offers customers an intangible logistics service rather than a specific product -"box". The concept of CC, can be used to build an integrated cloud logistics service platform (Li et al. 2013), to implement universal interconnectivity, logistics information sharing, and logistics tasks optimization distribution.

The rest of the paper is organised as follows. Section 2 reviews the literature related to this research. An overall architecture of smart box enabled product-service system for cloud logistics is presented in Section 3. Section 4 describes the PSS-based smart box enabled sustainable logistics service. Cloud logistics tasks dynamic optimization is described in Section 5. A case study is simulated in Section 6. Conclusions are drawn in Section 7.

\section{Literature review}

Three streams of literature are relevant to this research. They are PI, PSS and CC.

\subsection{The Physical Internet and its application in logistics}

The term "Physical Internet" is first introduced in 2006, and it raises many scholars' research interest and curiosity. An innovative logistics project of PI is launched by 
Laval University (Physical Internet Efficient Sustainable Logistics, 2010). Montreuil (2011) discusses the global logistics sustainable challenges and proposes PI as a new means to achieve the green logistics, e.g., world-standard smart green modular containers. Lin et al. (2015) develop a decomposition-based approach to formulate the selection of standard modular containers with the objectives of minimizing the total unused volume of containers. The applications of PI in logistics have been widely investigated, Sarraj et al. (2013) study the efficiency of an open and interconnected PI within the fast-moving consumer goods supply networks to improve transportation efficiency. One approach is drawing inspiration from the digital Internet to create a PI by using standardized "packets" and protocols for shipping (Wible et al., 2014). Sarraj et al. (2014) make an in-deep analysis of analogy between PI and the Digital Internet at the definition of interconnection, the structure of the network, and the routing of objectives through these networks. Ballot, Montreuil, and Thivierge (2012) present a functional design of PI facility: a road-rail hub, to provide an engineer a proof of concept. Pan et al. (2014) define the new research question related to inventory management in a PI Network and provide a view of how PI affects traditional inventory control policies.

\subsection{Product services system}

Product Service System (PSS) is a innovative business model that integrates services into products and furnishes the stakeholders with the relevant services rather than products. Mont (2002) clarifies the concept of PSS, outlines various approaches and trends towards the development of PSS and builds a theoretical framework. Tukker (2004) develops archetypical PSS business models, and the market and sustainability potential of the archetypical PSS models are evaluated. Geng et al. (2011) also present a systematic decision-making approach for PSS planning. These literature researches 
related to PSS provide a good support of concept, applied methods, and models for the further application in manufacturing fields. Williams (2007) provides a systematic overview of some existing and planned PSS initiatives in automobile industry. Huang et al. (2011) also discuss extending PSS concept to transform, elevate, and revitalize traditional equipment manufacturing industry such as Mold and Die sector. Boussier et al. (2011) develop a multi-agent-based logistics distribution network for urban goods delivery researching network management, organization and sharing. Ribeiro and Borsato (2014) propose the application of PSS practices into new product development processes, which makes companies provide sustainable products.

Sharing is also an important feature of PSS that has been used to provide good supports for sharing of services and products. Huang et al. (2012) apply the concept of PSS to design and develop a service-oriented framework called AUTOPS for sharing production information among automotive alliances. Zhang et al. (2015a) describe a framework of the Internet of Manufacturing Things to share the real-time manufacturing information, and a service encapsulation and virtualization access model to wrap the manufacturing services so that they can be easily reconfigured and reused (Zhang et al., 2015b). The relevant researches of RFID technology also describe the model and process of information acquisition, for example, principles and primary technologies of RFID (Want, 2006) and RFID-enable ubiquitous manufacturing and real-time interactions within production planning and scheduling level (Zhong et al., 2015a).

\subsection{Cloud computing for logistics tasks}

Currently, as the volume of logistics data is increasing exponentially, information processed to be meaningful is increasingly important for logistics tasks optimization distribution. The technology of CC is widely used in the logistics industry. Buyya et al. (2009) define CC and present the architecture for creating Clouds with market-oriented 
resource allocation. Li et al. (2013) study the resource virtualization and encapsulation of logistics resources in web services. $\mathrm{Hu}, \mathrm{Cao}$, and $\mathrm{Mu}$ (2013) present the conception of CC-based logistics vehicle monitoring platform. Motivated by the shop-floor uncertainty and complexity of typical mass-customization production companies, Zhong et al. (2013) develop an RFID-enabled real-time manufacturing execution system to track and trace manufacturing objects. Sun and Tao (2013) establish a regional logistics distribution centre location model which combines qualitative and quantitative analysis. Subramanian, Abdulrahman, and Zhou (2014) develop a conceptual model to analyze the short-term and long-term benefits of the integration of CC. Wang et al. (2014) indicate that logistics methods of resource matching are important problems of service resource management according to surveys on cloud logistics service. For sharing realtime location information and making an optimal decision, Zhang et al. (2014) propose a framework of a multi-agent based real-time production and logistics scheduling system, and a real-time optimization method for assigning shopfloor material handling tasks (Zhang et al. 2015c). Zhong et al. (2015b) introduce a Big Data approach for mining the invaluable trajectory knowledge from enormous RFID-enabled logistics data.

\section{Architecture of smart box enabled product-service system for cloud logistics}

An overall architecture of smart box enabled product-service system for cloud logistics (s-BPSS) is presented as Figure 1. It embraces three important elements at corresponding levels and aims to apply the technologies of PI, PSS, and CC into logistics tasks distribution and establish a referenced cloud logistics service platform to implement the global optimization for logistics tasks.

The bottom-level element is the hardware infrastructure encapsulated by PI. Key characteristics and technology of PI are applied to logistics tasks transportation, 
handling, storage and supply, provide the logistics with world-standard smart encapsulation agent and interaction transmission protocol, and facilitate the logistics to evolve towards PI. The most important characteristic of PI is the PI-container, which is physical packet/container used to encapsulate physical objects.

The middle-level element is smart box enabled product-service system. It is an innovative business model that meets users' requirements differentiating from traditional logistics service model. This model furnishes stakeholders with shared service of encapsulating goods in smart boxes, rather than a product. This smart box is similar to the PI-container on some key functional specifications.

The top-level element is a cloud logistics service platform integrated with advanced technologies. This level provides software service for the global optimization for cloud logistics. Under this framework, Logistic tasks management (LTM) is responsible for processing and standardizing the logistics tasks information. A real-time information driven dynamic optimization method (RTOM) is presented to implement logistics tasks assignment and loading. Real-time tracking and traceability of logistics tasks can also be achieved. Loading verification service (LVS) is designed to provide services of avoiding wrong loading of smart boxes and containers.

\# Figure 1. Architecture of smart box enabled product-service system for cloud logistics

\section{PSS-based smart box enabled sustainable logistics services}

Sharing is the key feature of s-BPSS. Consequently, it is an important that how the sharing of information, services, and products can be achieved in the logistics industry. PSS is an innovative model to realize the sharing approach (Huang et al., 2011). 
Smart box is designed to encapsulate goods, which is standard, smart, and recyclable. The key functional specifications of the designed smart box are similar to the PI-containers defined (Montreuil, 2011). They are:

- $\quad$ easy to flow through various transport, handling, and storage means;

- $\quad$ easy to handle, transport, seal, load, unload, compose, and decompose;

- made of environmentally-friendly materials with low cost;

- sealable for security purposes;

Nevertheless, the smart box has some characteristic functions differentiating from the PI-containers. To design suitable box with maximal space utilizations and universality, many investigations and surveys have been done on the e-business enterprises such as Alibaba, TAOBAO, and three kinds of dimensions of smart box are designed, namely small-sized, medium-sized and large-sized boxes, to meet the requirements for encapsulating goods. Box attached intelligent RFID tags has the capacity of identification, integrity, monitoring, tracking, traceability and location. The tag is an integrated circuit for storing and processing information, modulating/demodulating a radio-frequency signal.

To illustrate the role of PSS-based smart box in cloud logistics, a simplified logistics services scenario without losing generality is proposed as Figure 2, which composes of one shared warehouse, the 3PL, smart boxes, and customers. Figure 2 describes the working principle of the PSS-based smart box enabled logistics service.

\# Figure 2. The working principle of PSS-based smart box enabled logistics service The 3PL take the ownership of smart boxes and are responsible for the management of them, including box maintenance, status monitoring, information management, and recycling smart boxes. Thus, these boxes can be shared by all 
members among logistics enterprises. A shared warehouse is used to store these smart boxes.

The 3PL receive logistics distribution requests of goods from customers. Customer orders (COs) are encapsulated into the fitting smart boxes at the warehouse. Then, they are transported and delivered to customers. This smart box is recycled and stored at the warehouse for next time using. Customers pay fees for the 3PL. Consequently, smart boxes and that using packaging purpose can be considered as products, and service respectively. Logistics services such as transportation services, information services, and loading services, etc. are provided by the 3PL. The roles of stakeholders also are different from those in traditional logistics business model. Overall, PSS-based smart box enabled sustainable logistics service has more advantages

From an economical perspective, the total cost of a smart box includes: 1) the cost of raw material, manufacturing, maintenance; 2) the cost of a set of dedicated RFID devices. The former is $40 \mathrm{CNY}$, and the latter is $20 \mathrm{CNY}$. Statistical analysis reveals that the average maintenance cost of each used smart box once is about $0.5 \mathrm{CNY}$. The costs of a wood-made and a rectangular, stackable paperboard-made box are $25 \mathrm{CNY}$ and $0.8 \mathrm{CNY}$ respectively. Considering the total number of paper-made and wooden boxes widely, the average estimated cost of two kinds of boxes is $4 \mathrm{CNY}$. Comparison of smart and paper-made box on cost and use-pattern is made as Table 1. Supposing the average useful life of a smart box is at least 1000 recurrently, the total cost of using smart box is much less than that of using paper-made boxes. The saving cost of using smart box can be shared by stakeholders.

\# Table 1. Comparison of smart box and paper-made box

From an environmental perspective, the wood-made boxes used in current logistics usually are disposable. As a result, it is difficult to recycle. Production of 
paper-based boxes causes waste of natural resources, and emission of greenhouse gas. It can be demonstrated that smart box that is made of environmentally-friendly materials and recyclable is a green, low-carbon, and intelligent logistics packaging pattern.

From a manpower cost perspective, goods packaging is a labor-intensive industry. Manpower cost represents an increasing proportion in the total cost. In this business model, implementing above works just needs fewer workers with the supporting handled RFID readers. Thus, saving manpower resource, decreasing labor cost and manual errors can be also achieved.

\section{Logistics tasks dynamic optimization method}

A real-time information driven cloud logistics optimization method by introducing CC based on s-BPSS is presented as the top of Figure 1. It contains logistics tasks management and optimization loading service (OLS).

\subsection{Symbols}

Symbols used in this model are categorized into notations and decision variables.

\subsubsection{Notations}

$h_{i}^{\prime} \quad$ the length of the $i$-th COs, $i=\{1,2, \cdots, n\}$

$w_{i} \quad$ the length of the $i$-th COs

$h_{i} \quad$ the length of the $i$-th COs

$l_{j}^{\prime} \quad$ the length of the $j$-th smart box

$w_{i}^{\prime} \quad$ the width of the $j$-th smart box

$h_{i}^{\prime} \quad$ the height of the $j$-th smart box

$B_{j} \quad$ the $j$-th smart box, $j=(1,2,3)$. 


\subsubsection{Decision Variables}

$z_{J} \quad$ binary variable that equals 1 if container $J$ is selected, and 0 otherwise.

$\gamma_{i J}$ binary variable that equals 1 if $\operatorname{COs} i$ is loaded into container $J$, and 0 otherwise.

\subsection{Logistics tasks management}

LTM is responsible for managing logistics tasks, classifying and standardizing logistics information. Logistics tasks orders from customers can be divided into three classes. First class is offline order. Customers submit orders to the dedicated enterprises directly. The second is online. Customers log in an online account registered in the cloud platform and submit orders. The third is offline to online $(\mathrm{O} 2 \mathrm{O})$. Orders beyond the 3PL distribution capacity by offline are submitted to cloud platform, and subcontracted to the other. Above logistics tasks orders are defined as customer orders in the paper. 
A standardized COs information template integrated with EPCGlobal standard and RFID (GS1, 2003; Barchetti et al., 2009) is designed as Figure 3. It aims to standardize mixed and unordered information standards from different 3PL for the convenience of storage, sharing, and transmission.

\# Figure 3. A designed standardized COs information template

COs are classified in terms of address information. The address is divided into multiple administrative levels, namely nation, province, city, district, street, and detail address, as Figure 4. Based on the one-to-one mapping relationship between COs and address. A hierarchical tree-structure network of COs is founded as Figure 4. At the identical level with the same content of COs is classified as a class.

\# Figure 4. A hierarchical tree-structure network of COs

\subsection{The distance cluster of customer orders}

Then, a distance clustering for COs is described. First, definitions are given as follows: $d_{i j}$ and $D_{i j}$ denote the distance of samples (COs) $X_{i}$ and $X_{j}$, and class $G_{i}$ and $G_{j}$ respectively.

$$
D_{i j}=\min _{X_{i} \in G_{i}, X_{j} \in G_{j}} d_{i j}
$$

It is assumed that class $G_{p}$ and $G_{q}$ are grouped into a new class $G_{r}$, and the distance of an arbitrary class $G_{k}$ and $G_{r}$ is that:

$$
\begin{aligned}
D_{k r} & =\min _{X_{i} \in G_{k}, X_{j} \in G_{r}} d_{i j} \\
& =\min \left\{\min _{X_{i} \in G_{k}, X_{j} \in G_{p}} d_{i j}, \min _{X_{i} \in G_{k}, X_{j} \in G_{q}} d_{i j}\right\}
\end{aligned}
$$




$$
=\left\{D_{k p}, D_{k q}\right\}
$$

The procedure of the Distance Clustering as follows:

(1) Define distance of sample, calculate distance between two samples and get a new distance matrix $D_{(0)}$;

(2) Find out the shortest distance of element and set as $D_{p q}, G_{p}$ and $G_{q}$ are grouped into a new class $G_{r}$, namely $G_{r}=\left\{G_{p}, G_{q}\right\}$;

(3) Every new grouped class must be numbered in sequence;

(4) Calculate the distance between new class and the other in accordance with Equation (2);

(5) Repeat step (2), step (3) and step (4), and $r=r+1$;

(6) End up until all elements are grouped, if the number of the shortest distance element is more than one, all the corresponding elements are grouped into one class.

The elements at the right of a hierarchical tree-structure network of COs are grouped into an original clustering class. The distance of all elements in this class is zero. The process of clustering COs is a reverse clustering from right to left as shown in Figure 4. Then, clustering COs are called logistics orders (LOs). Loading smart boxes into containers with the objective of maximizing the total space of containers, subject to loading constraints, is studied. The schematic diagram of the loading process of smart boxes is designed as Figure 5.

\subsection{Dynamic optimization model of loading service}

Figure 5 describes that COs are grouped into LOs by clustering analysis. LOs are optimized to form a reasonable loading sequence and implement a high loading rate for 
containers (Mongeau and Bès, 2003; Lin et al., 2015). A mathematical model of RTOM for smart boxes loading problem is presented to implement the proposed goal. Notations are defined as follows.

$$
\begin{aligned}
& l_{i} \leq l_{1}^{\prime} \cap w_{i} \leq w_{1}^{\prime} \cap h_{i} \leq h_{1}^{\prime} \rightarrow i \in B_{1} \\
& l_{1}^{\prime}<l_{i} \leq l_{2}^{\prime} \cap w_{1}^{\prime}<w_{i} \leq w_{2}^{\prime} \cap h_{1}^{\prime}<h_{i} \leq h_{2}^{\prime} \rightarrow i \in B_{2} \\
& l_{2}^{\prime}<l_{i} \leq l_{3}^{\prime} \cap w_{2}^{\prime}<w_{i} \leq w_{3}^{\prime} \cap h_{2}^{\prime}<h_{i} \leq h_{3}^{\prime} \rightarrow i \in B_{3} \\
& M=\left(L_{J} \cdot W_{J} \cdot H_{J}\right) /\left(l_{3}^{\prime} \cdot w_{3}^{\prime} \cdot h_{3}^{\prime}\right) \\
& N=\left(L_{J} \cdot W_{J} \cdot H_{J}\right) /\left(l_{1}^{\prime} \cdot w_{1}^{\prime} \cdot h_{1}^{\prime}\right) \\
& M \leq z_{i}^{\lrcorner} \leq N \forall i, J \\
& \sum_{J} Z_{J} \leq n, \forall J \\
& \sum_{J} \sum_{i}{ }_{z_{i}^{J}} \leq \sum_{k} B_{k} \forall i, J, k \\
& Z_{J} \text { :binary, } \forall J \\
& \gamma_{i J} \text { :binary, } \forall i, J \\
& \text { Minimizing } \sum_{J} \sum_{i} Z_{J} \cdot\left(\left(L_{J} \cdot W_{J} \cdot H_{J}\right)-\stackrel{\wedge}{z_{i}^{J}} \cdot\left(l_{i}^{\prime} \cdot w_{i}^{\prime} \cdot h_{i}^{\prime}\right)\right) \\
& \text { Maximizing } \sum_{i} \gamma_{i J} \cdot\left(\stackrel{\wedge}{z_{i}^{J}} \cdot\left(l_{i}^{\prime} \cdot w_{i}^{\prime} \cdot h_{i}^{\prime}\right)\right.
\end{aligned}
$$


This model aims to assign and load LOs to containers such that the total unused volume of containers is minimal, as stated in (13), which is equivalent to maximizing the loading volume of each container, as equation (14). Information flow of COs and smart boxes enter into the order-box clustering and matching model as seen the third layer of Figure 1. The fitting smart box is selected to encapsulate goods, and form COsbox. The matching model of COs and smart box is represented as Equation (1)-(3), it denotes that if the dimension of COs meets the dimension of small-sized, mediumsized, and large-sized smart box, it can be dropped into $B_{1}, B_{2}, B_{3}$ respectively. Allowed loading number of COs in a container is limited within the range defined by (6)-(8). Constraint (9) denotes that the total number of the selected containers is less than all containers. Constraint (10) indicates that the available number of smart boxes is sufficient. Feasible values of decision variables are defined in (11) and (12).

\# Figure 5. The schematic diagram of process of smart boxes loading

The optimization method for loading smart box into containers with minimizing unused volume of containers is illustrated in Figure 6, where $G_{p}$ indicates that clustering of LOs, $G_{q}$ is the shortest distance of clustering for $G_{p}$, and they are clustered as a new clustering $G_{p q} . I_{0}$ is the total number of COs in $G_{p}\left(G_{p q}\right)$.

The procedure in Figure 6 is as follows. All COs are clustered to form LOs by a hierarchical tree-structure network. For original LOs, the quantity of COs in LOs $\left(G_{p}\right)$ is $I$ and initialized as 0 . If the dimension of container $J$ for COs is available, this COs is loaded into container, and the used and surplus volume of container $J$ is outputted and updated. If the quantity of COs in $G_{p}$ is less than or equal to $I_{0}$, then return to LOs $\left(G_{p}\right)$, else end up; Else, output the amount of COs of $J$ is $\stackrel{\wedge}{z_{i}^{J}}$, if $\stackrel{\wedge}{z_{i}^{J}}$ is less than $I_{0}$, then 
seeking for the shortest distance clustering $G_{q}$ for $G_{p}$, and form a new clustering $G_{p q}$, then to LOs $\left(G_{p}\right)$, else the end.

\# Figure 6. An optimization method for smart box loading

During the smart box loading, LVS is proposed to facilitate workers to load COs into the container exactly. Visual checking may cause errors due to a large quantity of COs, containers, similar letter printed on labels and worker's faults. This service can detect COs automatically through comparing loading information obtained by handheld RFID readers and that installed at container read tags, with original loading list. Only the two loading information is matched precisely can the COs be allowed to load. Otherwise, workers are alarmed. This service greatly eliminates wrong loading of smart boxes and containers.

\section{Case study}

A case study for logistics orders distribution of a distribution centre (DC) in a district is proposed to investigate the efficiency of presented RTOM. This district contains one DC and eight streets. They are numbered in sequence as 0 and $1,2, \ldots, 8$. The total number of COs is 731 in a period time. There are three types of smart boxes and a type of container, which are $0.45(\mathrm{~m}) \times 0.3(\mathrm{~m}) \times 0.3(\mathrm{~m}), 0.9(\mathrm{~m}) \times 0.6(\mathrm{~m}) \times 0.45(\mathrm{~m})$, $2(\mathrm{~m}) \times 1(\mathrm{~m}) \times 0.6(\mathrm{~m})$, and $4.02(\mathrm{~m}) \times 1.93(\mathrm{~m}) \times 1.9(\mathrm{~m})$ respectively.

By using above standardized COs, and order-box clustering and matching method, the information of $731 \mathrm{COs}$ is processed to form meaningful information shown as Table 2.

\# Table 2. The information of COs

Based on above hierarchical tree-structure network and the distance clustering analysis method, COs are grouped into eight original groups, and defined 
as $G_{1}, G_{2}, \ldots, G_{8}$ respectively, such as $G_{1}=(63,24,4)$. The results of clustering for all LOs is shown as Figure 7. Firstly, $G_{1}$ and $G_{8}$ are clustered into a new class $G_{9}\left(\left(G_{1}, G_{8}\right) \rightarrow\right.$ $\left.G_{9}\right) .\left(G_{3}, G_{4}\right) \rightarrow G_{10}$, and $\left(G_{6}, G_{7}\right) \rightarrow G_{11}$. Secondly, $\left(G_{5}, G_{10}\right) \rightarrow G_{12}$, and $\left(G_{2}, G_{11}\right) \rightarrow$ $G_{13}$. Finally, checking whether all COs have been assigned or not, and the logistics operations are taken concurrently.

\# Figure 7. Results of clustering for all LOs

A distribution network diagram for all COs is illustrated as Figure 8. Notation 0 presents DC, and 1, 2, 3, ., 8 denote eight streets. Solid line indicates the distribution route from DC to each street directly, and dashed line presents an optimal route. The number at the side of line is the distance of a point to another, and the number at brackets is the quantity of small, medium, and large COs respectively from left to right.

\# Figure 8. A distribution network diagram for COs

A distribution network diagram for all COs can be considered as a visualization of Figure 6. It can be found that original LOs within point 1 and 8 can be grouped as a new class. It is same to $(2,6,7)$ and $(3,4,5)$. The optimal distribution solutions for all COs (OS) are shown as Table 3.

\# Table 3. The optimal distribution solutions for all COs

A signal distribution method that is a one-to-one direct distribution between DC and address without sharing is universal. Current logistics distribution solutions for all COs (OS) are represented as Table 4.

\# Table 4. Current logistic distribution solutions for all COs

From Table 3, the first group includes two points: 1 and 8, the second is 7, 6, and 2 , and the third is 3, 4, and 5. For the first group, the loading content for all COs is 
divided into $(0,24,4)$ and $(180,21,1)$ optimally. The distribution route and the distribution distance of former are $0 \rightarrow 1 \rightarrow 0$ and $14 \mathrm{~km}$ respectively, and the latter are 0 $\rightarrow 1 \rightarrow 8 \rightarrow 0$ and $20.8 \mathrm{~km}$ and the surplus COs of point $1(63,0,0)$ and all COs of point 8 $(117,12,1)$ together are loaded into a container. The loading sequence of the latter is that, $(117,12,1)$ is loaded firstly, and $(63,0,0)$ is the second. Overall, the distribution frequency of the first group is 2, and the total distance is $34.8 \mathrm{~km}$. While from Table 4, the former is 3 , and the latter is $46.6 \mathrm{~km}$.

The comparison is made to illustrate advantages of RTOM. The total distribution frequency for all COs is 9 in OS, namely using 9 containers. While in CS, it is 13. The total distance of logistics distributions for all COs in OS is $93.4 \mathrm{~km}$, while it is $117.2 \mathrm{~km}$ in CS. The former saves 4 containers and decreases $23.8 \mathrm{~km}$ contrasted with the latter.

Figure 9 describes the average loading rate $(\mathrm{R})$ and the average loading rate per unit $\mathrm{KM}(\mathrm{R} / \mathrm{KM})$ for method 1 and 2 . $\mathrm{R}$ indicates that the total volume of all $\mathrm{COs}$ divides by the sum of the volume of all used containers. $\mathrm{R} / \mathrm{KM}$ denotes that the total volume of all COs divides by the total distance of delivering all COs. The higher the values of $\mathrm{R}$ and $\mathrm{R} / \mathrm{KM}$, the greater the efficiency of logistics distribution is. In Figure 9, 1 and 2 represent RTOM and traditional distribution method respectively. R/KM of 1 and 2 are 1.2019 and 0.9578 respectively. $\mathrm{R} / \mathrm{KM}$ of 1 is larger than that of 2 by 0.2441 , and the increased percentage of $\mathrm{R} / \mathrm{KM}$ of 1 compared to 2 is $25.48 \%$. $\mathrm{R}$ of 1 and 2 are 0.8504 and 0.5887 . $\mathrm{R}$ of 1 is larger than that of 2 by 0.2617 . $\mathrm{R}$ of 1 compared to 2 increases by $44.44 \%$.

The proposed method has advantages on increasing loading rate, reducing the total distribution distance, and optimising logistics resources. It is clear that the method 1 can increase significantly $\mathrm{R}$ for all $\mathrm{COs}$, and maximizing the space of containers. It 
can have positive effect on $\mathrm{R} / \mathrm{KM}$ for all $\mathrm{COs}$, which means that the total distribution distance for all COs has a conspicuous decrease. It should be pointed out that the number of COs is constant due to COs received in a period time. It leads to lower $\mathrm{R}$ for logistics distribution points 4,6 and 8 compared with others. It is a fact that the number of COs should be dynamic in a longer period time, thus R can be improved in a sense.

\# Figure 9. The average loading rate and the average loading rate per unit KM for method 1 and 2

\section{Conclusions}

In this paper, key issues and challenges related to cloud logistics orders distribution are discussed, especially in China. Three contributions are important in this research. The first contribution is that an innovative business model of PSS is adopted and collaborated with smart boxes to create a green, low-carbon logistics distribution pattern, and has the maximum revenue for all stakeholders. The second is that a PI-based and world-standard smart green box for encapsulating COs is designed to be recyclable, reduce the use of natural resources, and cost of logistics. The third is that the real-time information driven optimization method for logistics orders is proposed to increase the logistics efficiency (e.g., maximizing the volume of containers, optimising logistics loading and minimizing the total distribution).

Future work will focus on how to achieve revenue maximization with the most fitting quantity of smart boxes under meeting requirements of logistics tasks distribution. The number of inventory of smart box in logistics enterprise that meets encapsulation demand of logistics tasks and maintains the minimum inventory cost of smart boxes should also be investigated. In addition, how to change the man-dominant process of logistics distribution is an issue worth studying. 


\section{Acknowledgements}

This work was supported by the $<$ National Science Foundation of China $>$ under Grant $<51175435>$; $<$ Doctoral Fund of Ministry of Education of China $>$ under Grant $<20136102110022>$; $<111$ Project Grant of NPU $>$ under Grant $<$ B13044>.

\section{References}

Barchetti, U., A. Bucciero, M. De Blasi, L. Mainetti, and L. Patrono. 2009. "Implementation and testing of an EPCglobal-aware discovery service for itemlevel traceability." Proceedings of the International Conference on UltraModern Telecommunications and Workshops, pp. 1-8.

Boussier, J. M., T. Cucu, L. Ion, and D. Breuil. 2011. "Simulation of goods delivery process." International Journal of Physical Distribution \& Logistics Management 41(9): 913-930.

Ballot, E., B. Montreuil, and C. Thivierge. 2012. "Functional Design of Physical Internet Facilities: A Road-Rail Hub." In Progress in Material Handling Research: MHIA, Charlotte, NC (2012).

Buyya, R., C. S. Yeo, S. Venugopal, J. Broberg, and I. Brandic. 2009. "Cloud computing and emerging IT platforms: vision, hype, and reality for delivering computing as the 5th utility." Future Generation Computer Systems 25(6): 599616.

Geng, X., X. Chu, D. Xue, and Z. Zhang. 2011. "A systematic decision-making approach for the optimal product-service system planning." Expert Systems with Applications 38(9): 11849-11858.

GS1, 2003. EPCglobal. <http://www.gs1.org/epcglobal> (retrieved 01.01.13).

Hu, M., Y. Cao, and X. Mu. 2013. "Research of logistics vehicle monitoring platform based on cloud computing." LISS 2013, Springer: 359-364.

Huang, G. Q., T. Qu, R. Y. Zhong, Z. Li, H. D. Yang, Y. F. Zhang, and X. Chen. 2011. "Estabishing production service system and information collaboration platform for mold and die product." International Journal of Advance Manufacturing Technology 52(9-12): 1149-1160.

Huang, G. Q., T. Qu, Y. F. Zhang, and H. D. Yang. 2012. "RFID-enabled productservice system for automotive part and accessory manufacturing alliances." International Journal of Production Research 50(14): 3821-3840. 
Li, W., Y. Zhong, X. Wang, and Y. Cao. 2013. "Resource virtualization and service selection in cloud logistics." Journal of Network and Computer Applications 36(6): 1696-1704.

Lin, Y. H., R. D. Meller, K. P. Ellis, L. M. Thomas, and B. J. Lombardi. 2015. “A decomposition-based approach for the selection of standardized modular containers." International Journal of Production Research 52(15): 4660-4672.

Marston, S., Z. Li, S. Bandyopadhyay, J. Zhang, and A. Ghalsasi. 2011. "Cloud computing — The business perspective.” Decision Support Systems 51(1): 176189.

Mont, O. K. 2002. "Clarifying the concept of product-service system." Journal of cleaner production 10(3): 237-245.

Mongeau, M., and C. Bès. 2003. "Optimization of Aircraft Container Loading." IEEE Transactions on Aerospace and Electronic Systems 39 (1): 140-150.

Pan, S., M. Nigrelli, M. Ballot, R. Sarraj, and Y. Yang. 2014. "Perspectives of inventory control models in the Physical Internet: A simulation study." Computer \& Industrial Engineering 84: 122-132.

Physical Internet Efficient Sustainable Logistics, 2010, Retrieved April 20, 2013. http://www.physicalinternetinnitiative.org/.

Ribeiro, V. C., and M. Borsato. 2014. "Integrating Product-Service System Tools into New Product Development Processes." Journal of Integrated Design and Process Science 18(3): 3-18.

Sarraj, R., E. Ballot, S. Pan, D. Hakimi, and B. Montreuil. 2013. "Interconnected logistic network and protocols: simulation-based efficiencyassessment." International Journal of Production Research 52(11): 3185-3208.

Sarraj, R., E. Ballot, S. Pan, and B. Montreuil. 2014. "Analogies between Internet network and logistics service network: challenge involved in the interconnection.” Journal of Intelligent Manufacturing 25(6): 1207-1219.

Subramanian, N., M. D. Abdulrahman, and X. Zhou. 2014. "Integration of logistics and cloud computing service providers: cost and green benefits in the Chinese context." Transportation ResearchPart E: Logistics and Transportation Review 70: 86-98.

Sun, W., and L. Tao. 2013. "Cloud logistics mode-based location of regional logistics distribution center." Bridges 10: 9780784413159.9780784413360. 
The physical internet: a survey of logistics, The Economist, 2006, June, 17. The Economist Newspaper Limited, London

Tukker, A. 2004. "Eight types of product-service system: eight ways to sustainability? experiences from SusProNet." Business Strategy and the Environment 13(4): 246-260.

Want, R. 2006. “An introduction to RFID technology." Pervasive Computing, IEEE 5(1): 25-33.

Wang, J., X. Zhang, X. Hu, and J. Zhao. 2014. "Cloud logistics service mode and its several key issues." Journal of System and Management Sciences 4(2): 34-44.

Wible, B., J. Mervis, and N. S. Wigginton. 2014. "Rethinking the global supply chain." Science 34(6188): 1100-1103.

Williams, A. 2007. "Product service systems in the automobile industry: contribution to system innovation?" Journal of Cleaner Production 15(11-12): 1093-1103.

Zhang, Y. F., G. Zhang, J. Q. Wang, S. D. Sun, S. B. Si, and T. Yang. 2015a. "Realtime information capturing and integration framework of the internet of manufacturing things." International Journal of Computer Integrated Manufacturing 28(8): 811-822.

Zhang, Y. F., G. Q. Huang, S. D. Sun, and T. Yang. 2014. "Multi-agent based real-time production scheduling method for radio frequency identification enabled ubiquitous shopfloor environment." Computers \& Industrial Engineering 76: 89-97.

Zhang, Y. F., G. Zhang, Y. Liu, and D. Hu. 2015b. "Research on services encapsulation and virtualization access model of machine for cloud manufacturing." Journal of Intelligent Manufacturing. doi: 10.1007/s10845-015-1064-2.

Zhang, Y. F., G. Zhang, W. Du, J. Q. Wang, E. Ali, and S. D. Sun. 2015c. "An optimization method for shopfloor material handling based on real-time and multi-source manufacturing data." International Journal of Production Economics 165: 282-292.

Zhong, R. Y., Q. Y. Dai, T. Qu, G. J. Hu, and G. Q., Huang. 2013. "RFID-enabled realtime manufacturing execution system for mass-customization production." Robotics and computer-integrated manufacturing 29(2):283-292.

Zhong, R. Y., G. Q. Huang, S. Lan, Q. Dai, T. Zhang, and C. Xu. 2015a. “A two-level advanced production planning and scheduling model for RFID-enabled 
ubiquitous manufacturing." Advanced Engineering Informatics. doi:10.1016/j.aei.2015.01.002.

Zhong, R. Y., G. Q. Huang, S. Lan, Q. Y. Dai, X. Chen, and T. Zhang, 2015b. “A big data approach for logistics trajectory discovery from RFID-enabled production data.” International Journal of Production Economics 165: 260-272. 


\section{Tables}

Table 1. Comparison of smart box and paper-made box

Table 2. The information of COs

Table 3. The optimal distribution solutions for all COs

Table 4. Current logistic distribution solutions for all COs

\section{Figures}

Figure 1. Architecture of smart box enabled product-service system for cloud logistics

Figure 2. The working principle of PSS-based smart box enabled logistics service

Figure 3. A designed standardized COs information template

Figure 4. A hierarchical tree-structure network of COs

Figure 5. The schematic diagram of process of smart boxes loading

Figure 6. An optimization method for smart box loading

Figure 7. Results of clustering for all LOs

Figure 8. A distribution network diagram for $\mathrm{COs}$

Figure 9. The average loading rate and the average loading rate per unit KM for method 1 and 2 
Table 1. Comparison of smart box and paper-made box

\begin{tabular}{cccccc}
\hline Container Type & $\begin{array}{c}\text { Original } \\
\text { Cost/yuan }\end{array}$ & Maintenance & Number of & Total & Usage \\
Cost/yuan & use & Cost/yuan & Mode \\
Smart Box & 60 & 0.5 & 10000 & 5060 & Recyclable \\
Paper- & 4 & 0 & 10000 & 40000 & Disposable \\
made/Wooden Box & & & & & \\
\hline
\end{tabular}

Table 2. The information of COs

\begin{tabular}{cccccccc}
\hline $\begin{array}{c}\text { Street } \\
\text { type }\end{array}$ & $\begin{array}{c}\text { Total } \\
\text { number } \\
\text { of COs }\end{array}$ & $\begin{array}{c}\text { Quantity } \\
\text { of small } \\
\text { COs }\end{array}$ & $\begin{array}{c}\text { Quantity } \\
\text { of } \\
\text { medium } \\
\text { COs }\end{array}$ & $\begin{array}{c}\text { Quantity } \\
\text { of large } \\
\text { COs }\end{array}$ & $\begin{array}{c}\text { Quantity of } \\
0.45 \times 0.3 \\
\times 0.3 \text { box }\end{array}$ & $\begin{array}{c}\text { Quantity of } \\
0.45 \times 0.6 \\
\times 0.9 \text { box }\end{array}$ & $\begin{array}{c}\text { Quantity of } \\
0.9 \times 1 \times 2 \\
\text { box }\end{array}$ \\
\hline 1 & 91 & 63 & 24 & 4 & 63 & 24 & 4 \\
2 & 76 & 20 & 50 & 6 & 20 & 50 & 6 \\
3 & 72 & 50 & 20 & 2 & 50 & 20 & 2 \\
4 & 87 & 40 & 45 & 2 & 40 & 45 & 2 \\
5 & 109 & 100 & 6 & 2 & 100 & 6 & 3 \\
6 & 78 & 35 & 40 & 3 & 35 & 40 & 1 \\
7 & 88 & 67 & 2 & 1 & 67 & 20 & 12 \\
8 & 130 & 117 & 12 & 1 & 117 & 12 & 22 \\
Total & 731 & 492 & 217 & 22 & 492 & & 217 \\
\hline
\end{tabular}

Table 3. The optimal distribution solutions for all COs

\begin{tabular}{ccccc}
\hline \multicolumn{2}{c}{ Street Type } & Loading Content & Route & Distance \\
\hline \multirow{2}{*}{1} & 1 & $(0,24,4)$ & $0 \rightarrow 1 \rightarrow 0$ & 14 \\
& 8 & $(180,12,1)$ & $0 \rightarrow 1 \rightarrow 8 \rightarrow 0$ & 20.8 \\
\hline \multirow{2}{*}{2} & 7 & $(72,20,3)$ & $0 \rightarrow 7 \rightarrow 6 \rightarrow 0$ & 8.2 \\
& 6 & $(0,40,1)$ & $0 \rightarrow 6 \rightarrow 0$ & 4.6 \\
& 2 & $(50,16,4),(0,34,2)$ & $0 \rightarrow 6 \rightarrow 2 \rightarrow 0,0 \rightarrow 2 \rightarrow 0$ & 16.4 \\
\hline \multirow{2}{*}{3} & 3 & $(72,20,3)$ & $0 \rightarrow 4 \rightarrow 3 \rightarrow 0$ & 11.2 \\
& 4 & $(0,40,1)$ & $0 \rightarrow 4 \rightarrow 0$ & 5.4 \\
& 5 & $(118,11,2)$ & $0 \rightarrow 4 \rightarrow 5 \rightarrow 0$ & 12.8 \\
\hline
\end{tabular}

Table 4. Current logistic distribution solutions for all COs

\begin{tabular}{cccc}
\hline Street Type & Loading Content & Route & Distance \\
\hline 1 & $(63,24,4)$ & $0 \rightarrow 1 \rightarrow 0$ & $28(2)$ \\
2 & $(20,50,6)$ & $0 \rightarrow 2 \rightarrow 0$ & $21(3)$ \\
3 & $(50,20,2)$ & $0 \rightarrow 3 \rightarrow 0$ & $11.4(1)$ \\
4 & $(40,45,2)$ & $0 \rightarrow 4 \rightarrow 0$ & $10.8(2)$ \\
5 & $(100,6,3)$ & $0 \rightarrow 5 \rightarrow 0$ & $11(1)$ \\
6 & $(35,40,3)$ & $0 \rightarrow 6 \rightarrow 0$ & $9.2(2)$ \\
7 & $(67,20,1)$ & $0 \rightarrow 7 \rightarrow 0$ & $7.2(1)$ \\
8 & $(117,12,1)$ & $0 \rightarrow 8 \rightarrow 0$ & $18.6(1)$ \\
\hline
\end{tabular}




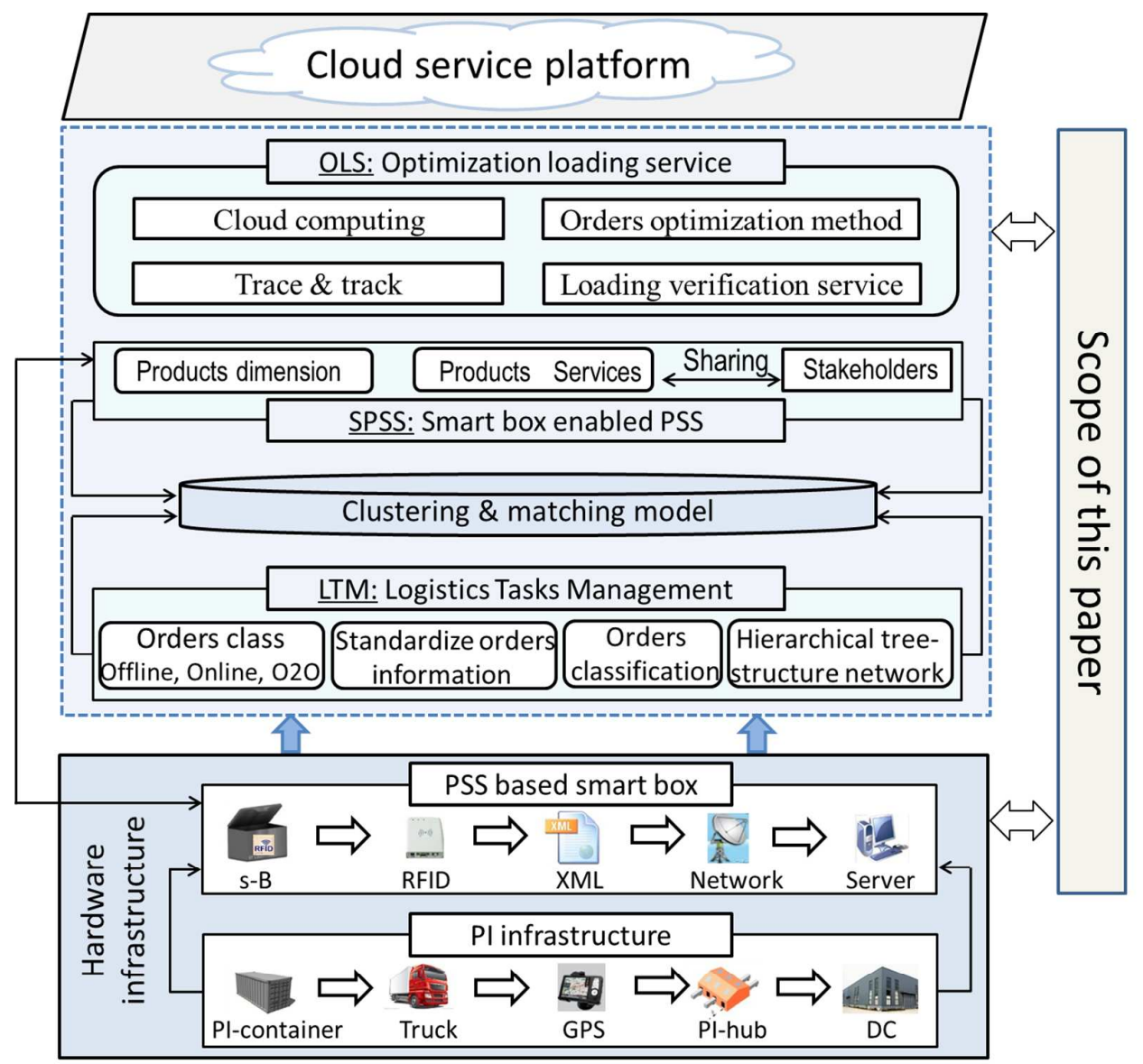

Figure 1. Architecture of smart box enabled product-service system for cloud logistics

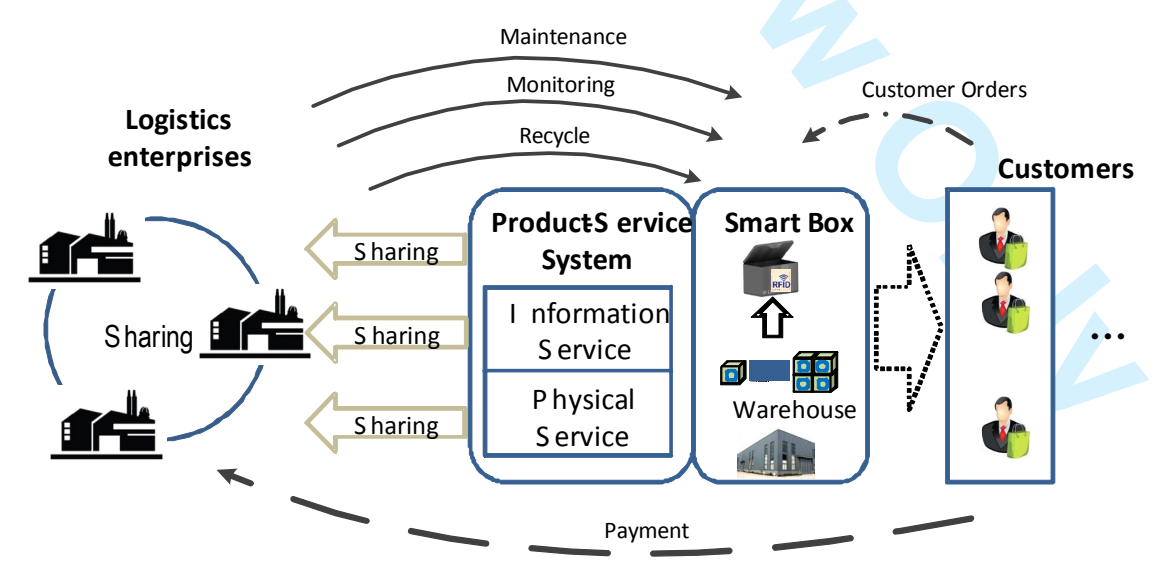

Figure 2. The working principle of PSS-based smart box enabled logistics service 


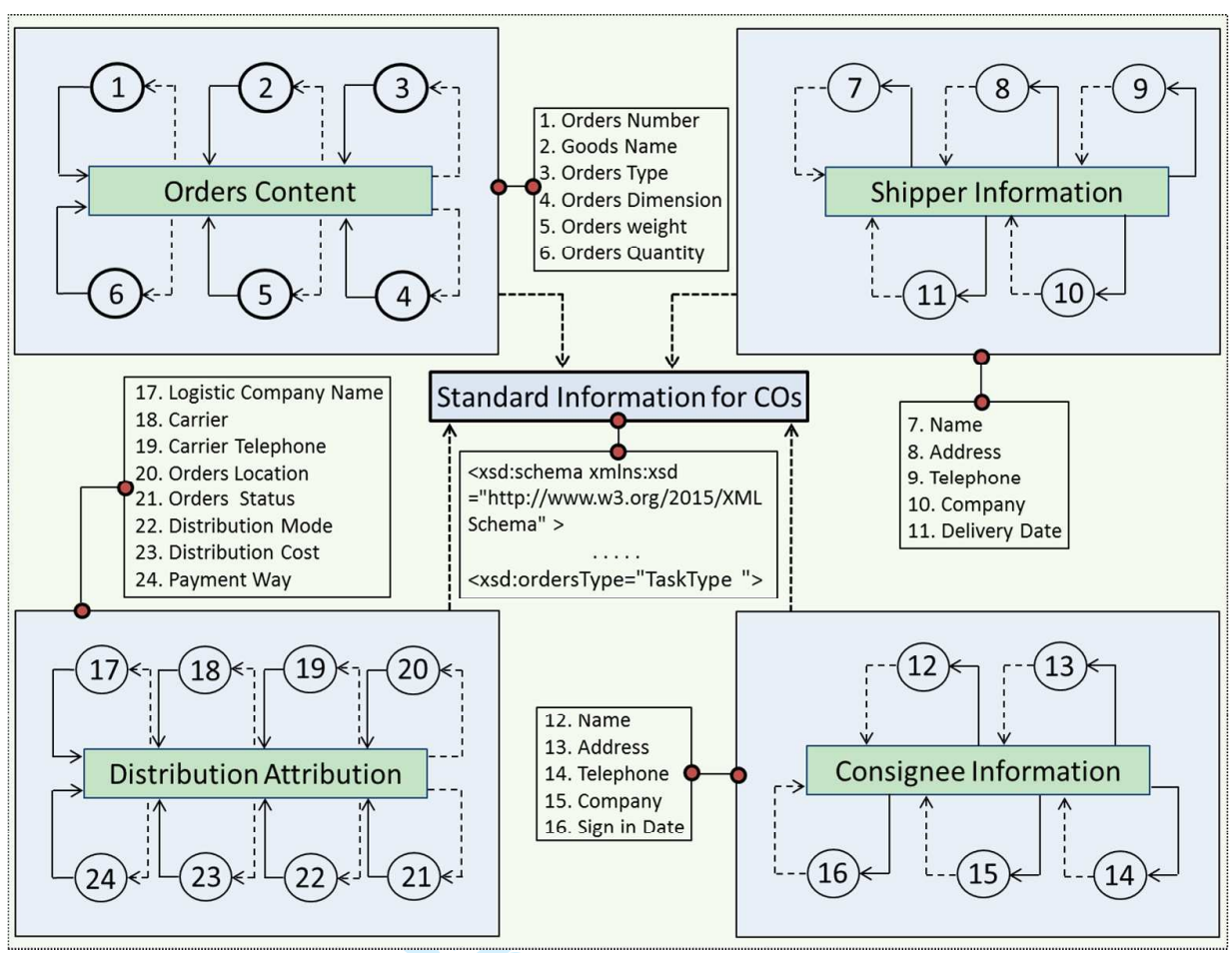

Figure 3. A designed standardized COs information template

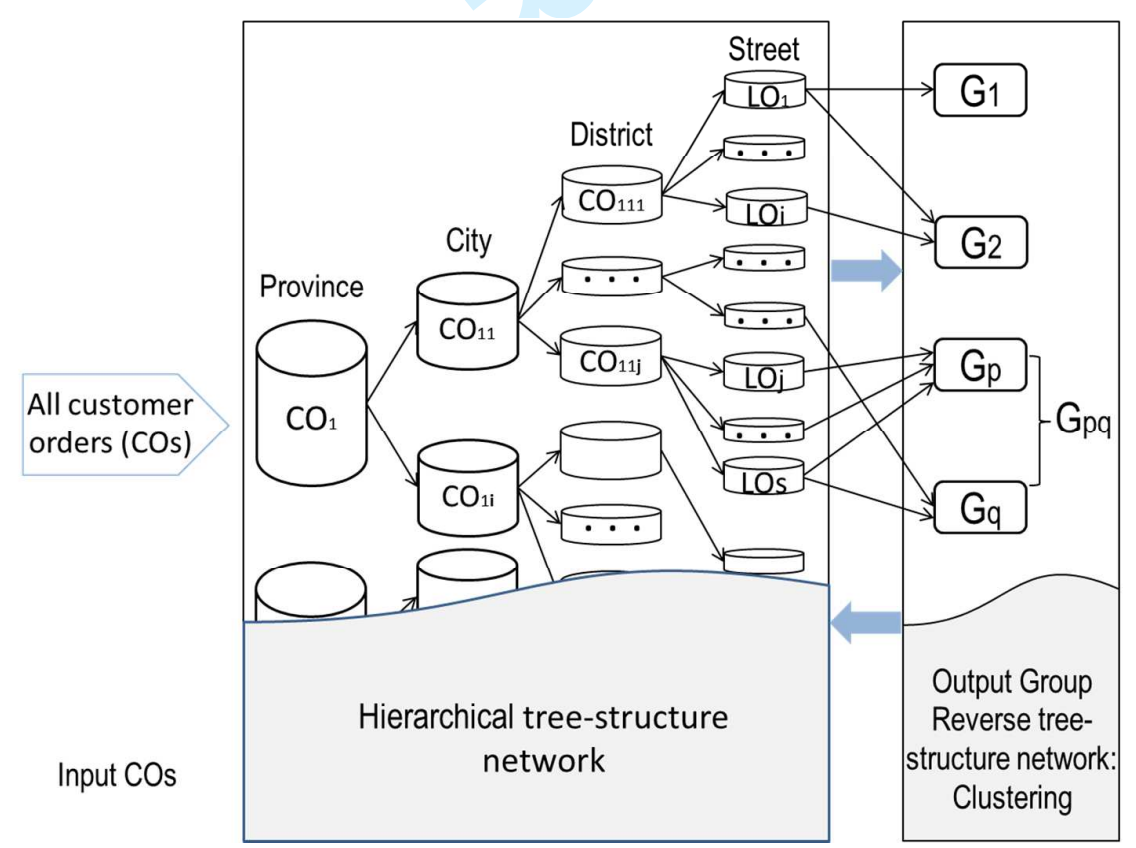

Figure 4. A hierarchical tree-structure network of COs 


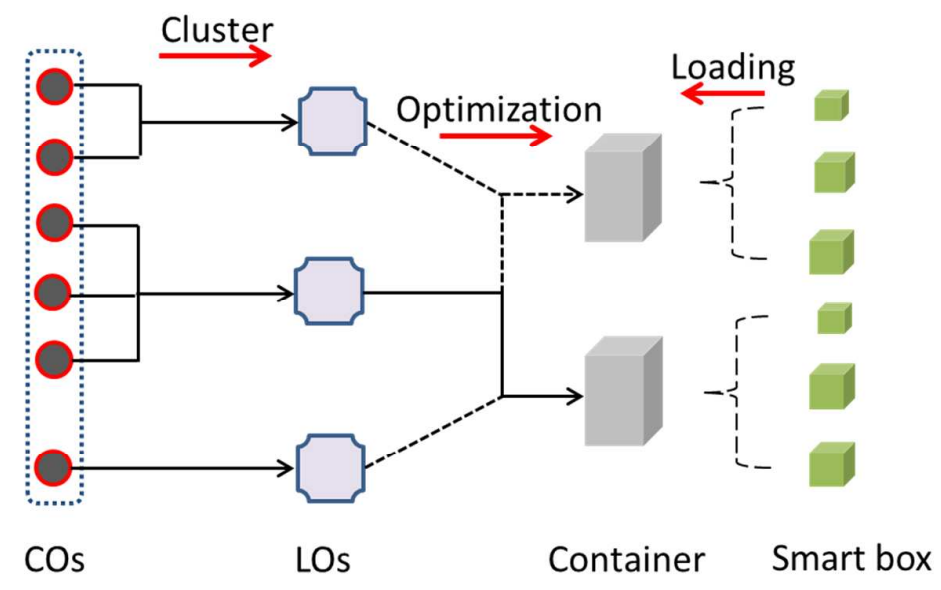

Figure 5. The schematic diagram of process of smart boxes loading

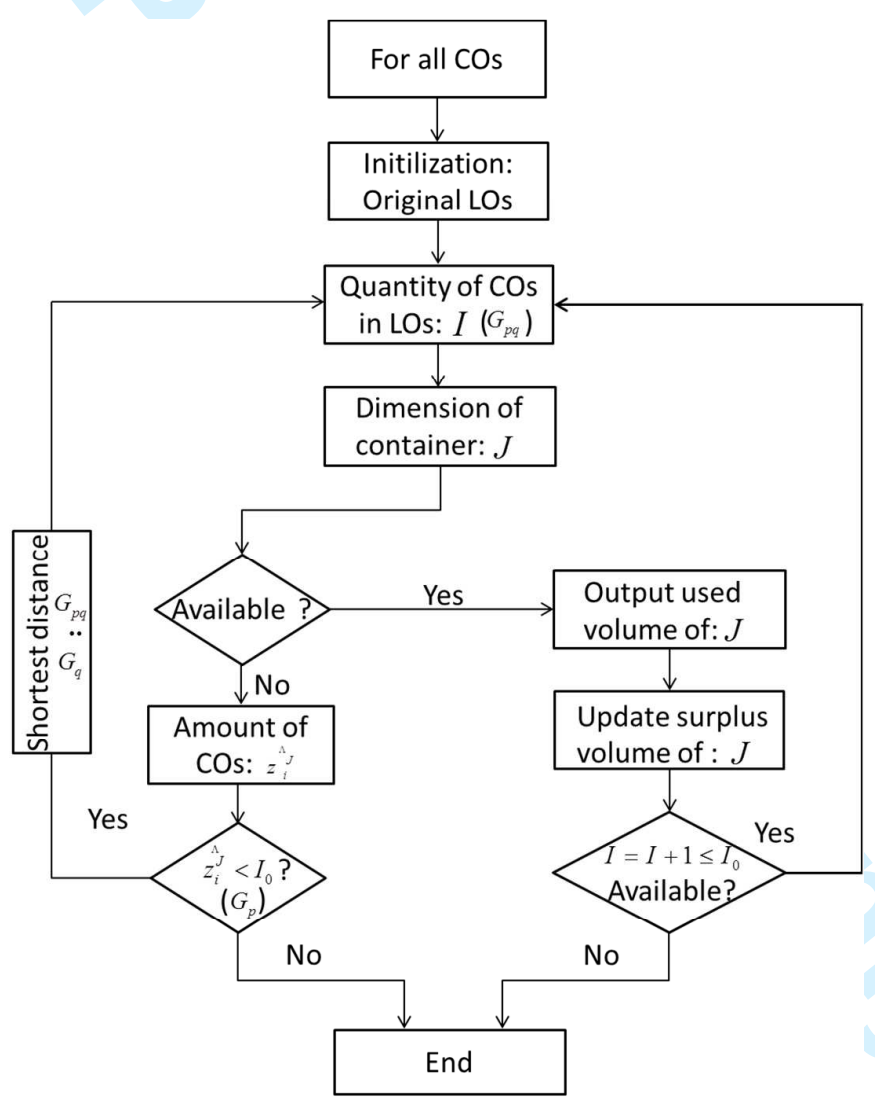

Figure 6. An optimization method for smart box loading 


1
2
3
4
5
6
7
8
9
10
11
12
13
14
15
16
17
18
19
20
21
22
23
24
25
26
27
28
29
30
31
32
33
34
35
36
37
38
39
40
41
42
43
44
45
46
47
48
49
50
51
52
53
54
55
56
57
58
60

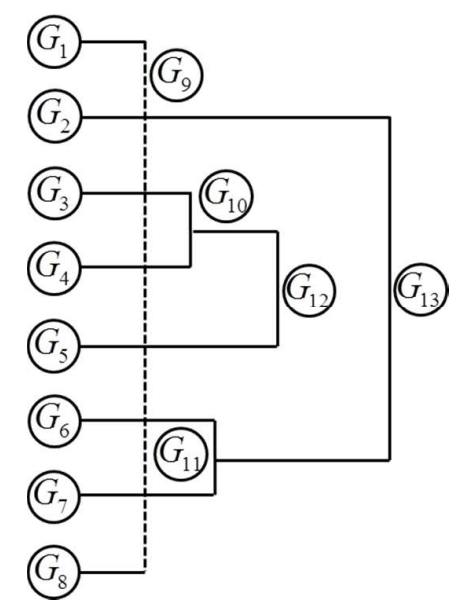

Figure 7. Results of clustering for all LOs

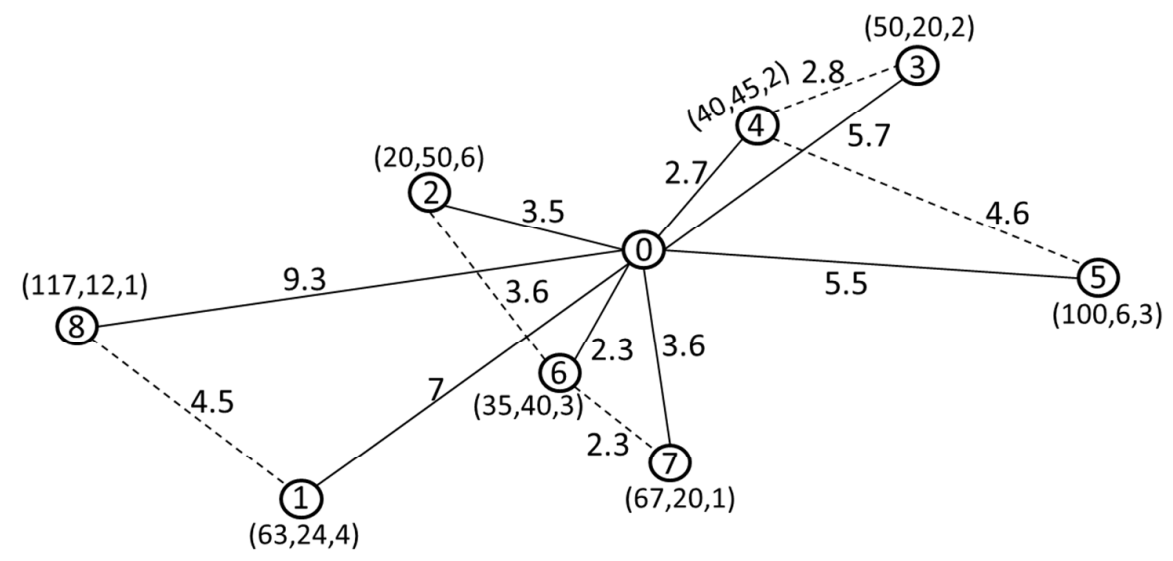

Figure 8. A distribution network diagram for $\mathrm{COs}$ 
Figure 9. The average loading rate and the average loading rate per unit KM for method 1 and 2

1

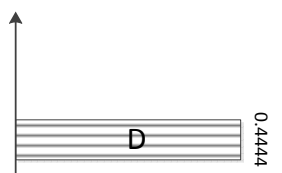

2
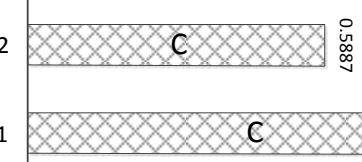

A Average loading

rate per unit KM
Increased percentage of

B average loading rate per unit

Q Average loading rate

D Increased percentage of coloading rate of 1: Proposed optimisation method 2: Current universal method

1

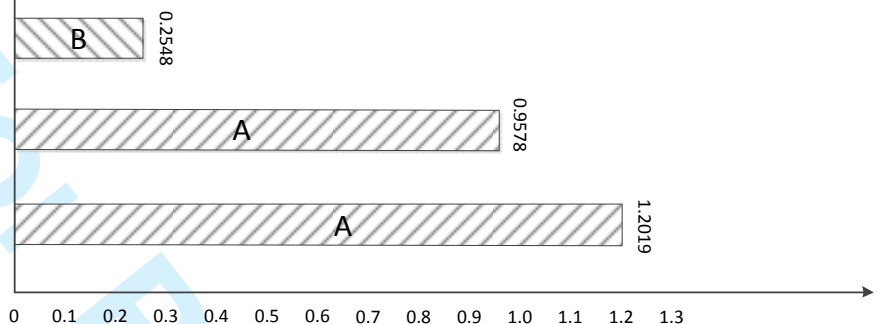

\section{1 and 2}

http://mc.manuscriptcentral.com/tprs Email: ijpr@tandf.co.uk 